\title{
Gravitational WAVES ABOUt CURVED BACKGROUndS: A CONSISTENCY ANALYSIS IN DE SitTER SPACETIME
}

\author{
Donato Bini, ${ }^{1,2}$ Salvatore Capozziello, ${ }^{3,4}$ Giampiero Esposito ${ }^{4}$ \\ ${ }^{1}$ CNR, Istituto per le Applicazioni del Calcolo "M. Picone", \\ Via del Policlinico 137, 00161 Rome, Italy \\ ${ }^{2}$ International Center for Relativistic Astrophysics - I.C.R.A., \\ University of Rome "La Sapienza," 00185 Rome, Italy \\ ${ }^{3}$ Dipartimento di Scienze Fisiche, Complesso Universitario di Monte S. Angelo, \\ Via Cintia, Edificio 6, 80126 Napoli, Italy \\ ${ }^{4}$ Istituto Nazionale di Fisica Nucleare, Sezione di Napoli, \\ Complesso Universitario di Monte S. Angelo, \\ Via Cintia, Edificio 6, 80126 Napoli, Italy
}

(Dated: May 21, 2018)

\begin{abstract}
Gravitational waves are considered as metric perturbations about a curved background metric, rather than the flat Minkowski metric since several situations of physical interest can be discussed by this generalization. In this case, when the de Donder gauge is imposed, its preservation under infinitesimal spacetime diffeomorphisms is guaranteed if and only if the associated covector is ruled by a second-order hyperbolic operator which is the classical counterpart of the ghost operator in quantum gravity. In such a wave equation, the Ricci term has opposite sign with respect to the wave equation for Maxwell theory in the Lorenz gauge. We are, nevertheless, able to relate the solutions of the two problems, and the algorithm is applied to the case when the curved background geometry is the de Sitter spacetime. Such vector wave equations are studied in two different ways: i) an integral representation, $i i$ ) through a solution by factorization of the hyperbolic equation. The latter method is extended to the wave equation of metric perturbations in the de Sitter spacetime. This approach is a step towards a general discussion of gravitational waves in the de Sitter spacetime and might assume relevance in cosmology in order to study the stochastic background emerging from inflation.
\end{abstract}




\section{INTRODUCTION}

One of the longstanding problems of modern gravitational physics is the detection of gravitational waves, for which the standard theoretical analysis relies upon the split of the space-time metric $g_{a b}$ into "background plus perturbations", i.e.

$$
g_{a b}=\gamma_{a b}+h_{a b}
$$

where $\gamma_{a b}$ is the background Lorentzian metric, often taken to be of the Minkowski form $\eta_{a b}$, while the symmetric tensor field $h_{a b}$ describes perturbations about $\gamma_{a b}$. However, the background $\gamma_{a b}$ needs not to be Minkowskian in several cases of physical interest, nor it has to be always a solution of the vacuum Einstein equations. As a consequence, we are therefore aiming to investigate in more detail what happens if the background space-time $\left(M, \gamma_{a b}\right)$ has a non-vanishing Riemann curvature.

This issue has to be seriously considered from an experimental point of view since the gravitational wave detectors of new generation are designed also to investigate strong-field regimes: this means that the physical situations, where only the standard Minkowski background is taken into account, could be misleading in order to achieve self-consistent results.

In particular, several ground-based laser interferometers have been built in the United States (LIGO) [1], Europe (VIRGO and GEO) [2, 3], and Japan (TAMA) [4] and are now in the data taking phase for frequency ranges about $10^{-1} k H z$. However, new advanced optical configurations allow to reach sensitivities slightly above and below the standard quantum limit for free test-particles, hence we are now approaching the epoch of second [5] and third [6] generation of gravitational wave detectors. This fact, in principle, allows to investigate wide ranges of frequencies where strong field regimes or alternative theories of gravity can be considered [7, 8, 9]. Besides, the laser interferometer space antenna (LISA) [10] (which is mainly devoted to work in the range $10^{-4} \sim 10^{-2} \mathrm{~Hz}$ ) should fly within the next decade principally aimed at investigating the stochastic background of gravitational waves. At much lower frequencies $\left(10^{-17} \mathrm{~Hz}\right)$, cosmic microwave background (CMB) probes, like the forthcoming PLANCK satellite, are designed to detect also gravitational waves by measuring the CMB polarization [11] while millisecond pulsar timing can set interesting upper limits in the frequency range between $10^{-9} \sim 10^{-8} \mathrm{~Hz}$ [12]. At these frequencies, the large number of millisecond pulsars detectable by the square kilometer array would provide 
a natural ensemble of clocks which can be used as multiple arms of a gravitational wave detector [13].

This forthcoming experimental situation is intriguing but deserves a serious theoretical analysis which cannot leave aside the rigorous investigation of strong-field regimes and the possibility that further polarization states of gravitational waves could come out in such regimes. For example, if one takes into account scalar-tensor theories of gravity [7] or higher-order theories [8], scalar-massive gravitons should be considered. This implies that the standard approach where gravitational waves are assumed as small perturbations (coming only from Einstein's general relativity) on a Minkowski background could be totally insufficient. On the other hand, the existence of these further polarization modes could be a straightforward solution of the dark matter problem since massive gravitons could be testable cold dark matter candidates as discussed in [14, 15].

In this paper, we want to face the issue of the rigorous formulation of gravitational wave problem in curved backgrounds. In particular, we want to perform a consistency analysis of gravitational waves in the de Sitter spacetime. Achieving solutions in this maximally symmetric background could constitute the paradigm to investigate any curved spacetime by the same techniques and could have interesting cosmological applications if a conformal analysis is undertaken as, for example in [9], where it is shown how the amplitude of cosmological gravitational waves strictly depends on the cosmological background.

It is straightforward to show that, in a covariant formulation, the supplementary condition for gravitational waves can be described by a functional $\Phi_{a}$ acting on the space of symmetric rank-two tensors $h_{a b}$ occurring in Eq. (1). For any choice of $\Phi_{a}$, one gets a different realization of the invertible operator $P_{a b}{ }^{c d}$ on metric perturbations. The basic equations of the theory read therefore as

$$
\begin{gathered}
P_{a b}{ }^{c d} h_{c d}=0, \\
\Phi_{a}(h)=0,
\end{gathered}
$$

where $P_{a b}^{c d}$ results from the expansion of the action functional to quadratic order in the metric perturbations. A deep link exists between classical and quantum theory, since in the latter, the one-loop analysis depends on the functional determinant of $P_{a b}{ }^{c d}$, after requiring that all metrics in Eq. (1) are positive-definite, i.e. Riemannian. Our analysis will instead be Lorentzian and classical. 
The layout of the paper is the following. Section 2 studies the de Donder choice for $\Phi_{a}$ and its preservation, while Secs. 3 and 4 deal with massless Green functions in de Sitter spacetime. This is done because the problem of preserving Eq. (3) under infinitesimal diffeomorphisms leads precisely to vector wave equations. These are solved by an integral representation or by separation of variables. This analysis prepares the ground for studying the wave equation on metric perturbations itself through separation of variables, in Sec. 6 . Concluding remarks and open problems are presented in Sec. 7, while relevant details are given in the Appendix.

\section{PRESERVATION OF THE DE DONDER SUPPLEMENTARY CONDITION}

Our first concern is how to implement in a consistent way the choice of supplementary condition. In general relativity, this is taken to be of the de Donder type (below $h \equiv \gamma^{c d} h_{c d}$ )

$$
\Phi_{a}(h)=\nabla^{b}\left(h_{a b}-\frac{1}{2} \gamma_{a b} h\right)
$$

if one wants to obtain the standard covariant wave operator on metric perturbations, where $\nabla^{b}$ denotes covariant derivative with respect to the background metric $\gamma_{a b}$. Under infinites-

imal space-time diffeomorphisms, the metric perturbations suffer the variation (the round brackets denoting symmetrization)

$$
\delta h_{a b}=\nabla_{(a} \varphi_{b)}
$$

where $\varphi_{b}$ is a covector, with associated one-form $\varphi_{b} d x^{b}$ and vector field $\varphi^{a} \frac{\partial}{\partial x^{a}}$ (having set $\varphi^{a} \equiv \gamma^{a b} \varphi_{b}$, which results from the isomorphism between tangent and cotangent space to the background space-time, that turns covectors into vectors, or the other way around). The change suffered from the de Donder gauge in (4) when metric perturbations are varied according to (5) is then found to be

$$
\delta \Phi_{a}(h)=-\left(\delta_{a}^{b} \square+R_{a}^{b}\right) \varphi_{b}
$$

where $\square$ is the standard d'Alembert operator in curved space-time, i.e. $\square \equiv \gamma^{c d} \nabla_{c} \nabla_{d}$. By virtue of Eqs. (4) and (6), if the de Donder gauge was originally satisfied, it is preserved under space-time diffeomorphisms if and only if $\varphi_{b}$ solves the equation $\delta \Phi_{a}(h)=0$. At this stage, to fully exploit what is known about the wave equation for Maxwell theory in curved 
space-time in the Lorenz gauge ${ }^{1}$ we bear in mind that this reads as

$$
\left(-\delta_{a}^{b} \square+R_{a}^{b}\right) A_{b}=0
$$

This suggests adding $R_{a}^{b} \varphi_{b}$ to both sides of $\delta \Phi_{a}(h)=0$ (see (6)), so as to cast it eventually in the form

$$
P_{a}^{b} \varphi_{b}=2 R_{a}^{b} \varphi_{b}
$$

where

$$
P_{a}^{b} \equiv-\delta_{a}^{b} \square+R_{a}^{b}
$$

is the standard gauge-field operator (see round brackets in Eq. (7)) in the Lorenz gauge. For this operator, the inverse $\widetilde{P}_{a}^{b}$ is an integral operator with kernel given by the photon Green function, so that we can solve Eq. (8) in the form

$$
\varphi_{c}=\varphi_{c}^{(0)}+2 \widetilde{P}_{c}^{a} R_{a}^{b} \varphi_{b}
$$

where $\varphi_{c}^{(0)}$ is a solution of the homogeneous wave equation [17]

$$
P_{a}^{b} \varphi_{b}^{(0)}=0
$$

while $\widetilde{P}_{c}^{a}$ is the inverse operator, satisfying

$$
\widetilde{P}_{c}^{a} P_{a}^{b}=\delta_{c}^{b}
$$

This $\widetilde{P}_{c}^{a}$ is an integral operator with kernel given by the massless spin-1 Green function $G_{a b}\left(x, x^{\prime}\right) \equiv G_{a b^{\prime}}$. The latter can be chosen, for example, to be of the Feynman type, i.e. that solution of the equation (see Appendix for the notation)

$$
\left(-\delta_{a}^{b} \square+R_{a}^{b}\right) G_{b c^{\prime}}=g_{a c^{\prime}} \frac{\delta\left(x, x^{\prime}\right)}{\sqrt{-\gamma}},
$$

having the asymptotic expansion as $\sigma \rightarrow 0$ [18, 19]

$$
G_{a b^{\prime}} \sim \frac{\mathrm{i}}{8 \pi^{2}}\left[\sqrt{\triangle} \frac{g_{a b^{\prime}}}{(\sigma+\mathrm{i} \varepsilon)}+V_{a b^{\prime}} \log (\sigma+\mathrm{i} \varepsilon)+W_{a b^{\prime}}\right],
$$

where $\sigma\left(x, x^{\prime}\right)$ is the Ruse-Synge world function [20, 21, 22], equal to half the square of the geodesic distance $\mu$ between the points $x$ and $x^{\prime}$.

\footnotetext{
${ }^{1}$ In [16], the author L. Lorenz, who was studying the identity of the vibrations of light with electrical currents, built a set of retarded potential for electrodynamics which, with hindsight, can be said to satisfy the gauge condition $\nabla^{\mu} A_{\mu}=0$, which therefore should not be ascribed to H. Lorentz.
} 


\section{MASSLESS GREEN FUNCTIONS IN DE SITTER SPACETIME}

This general scheme can be completely implemented in the relevant case [23] of de Sitter space where, relying upon the work in [24], we know that the massless spin-1 Green function reads as

$$
G_{a b^{\prime}}=\alpha(\mu) g_{a b^{\prime}}+\beta(\mu) n_{a} n_{b^{\prime}}
$$

where $\mu\left(x, x^{\prime}\right) \equiv \sqrt{2 \sigma\left(x, x^{\prime}\right)}$ is the geodesic distance between $x$ and $x^{\prime}, n^{a}\left(x, x^{\prime}\right)$ and $n^{a^{\prime}}\left(x, x^{\prime}\right)$ are the unit tangents to the geodesic at $x$ and $x^{\prime}$, respectively, for which

$$
n_{a}\left(x, x^{\prime}\right)=\nabla_{a} \mu\left(x, x^{\prime}\right), n_{a^{\prime}}\left(x, x^{\prime}\right)=\nabla_{a^{\prime}} \mu\left(x, x^{\prime}\right)
$$

while, in terms of the variable

$$
z \equiv \frac{1}{2}\left(1+\cos \frac{\mu}{\rho}\right)
$$

the coefficient functions $\alpha$ and $\beta$ are given, in four dimensions, by [24]

$$
\begin{gathered}
\alpha(z)=\frac{1}{48 \pi^{2} \rho^{2}}\left[\frac{3}{(1-z)}+\frac{1}{z}+\left(\frac{2}{z}+\frac{1}{z^{2}}\right) \log (1-z)\right], \\
\beta(z)=\frac{1}{24 \pi^{2} \rho^{2}}\left[1-\frac{1}{z}+\left(\frac{1}{z}-\frac{1}{z^{2}}\right) \log (1-z)\right] .
\end{gathered}
$$

Strictly speaking, the formulae (18)-(19) are first derived in the Euclidean de Sitter space. In the Lorentzian de Sitter spacetime $M$ which is what we are interested in, one can define the set [24]

$$
J_{x} \equiv\left\{x^{\prime} \in M: \exists \text { geodesic from } x \text { to } x^{\prime}\right\}
$$

Moreover, it is well-known that $M$ can be viewed as an hyperboloid imbedded in flat space, i.e. as the set of points $Y^{a} \in \mathbf{R}^{n+1}$ such that

$$
Y^{a} Y^{b} \eta_{a b}=\rho^{2}
$$

where $\eta_{a b}=\operatorname{diag}(-1,1, \ldots, 1)$, so that its induced metric reads as

$$
d s^{2}=\eta_{a b} d Y^{a} d Y^{b}
$$

As is stressed in Ref. [24], the relation

$$
z\left(x, x^{\prime}\right)=\frac{1}{2}\left[1+\frac{\eta_{a b} Y^{a}(x) Y^{b}\left(x^{\prime}\right)}{\rho^{2}}\right]
$$


is well defined both inside and outside $J_{x}$, and it is an analytic function of the coordinates $Y^{a}$. Thus, Eq. (23) makes it possible to define $z\left(x, x^{\prime}\right)$ everywhere on de Sitter, and one can define the geodesic distance

$$
\mu\left(x, x^{\prime}\right) \equiv 2 \rho \cos ^{-1}(\sqrt{z})
$$

as the limiting value [24] above the standard branch cut of $\cos ^{-1}$. Along similar lines, the equations defining $n_{a}, n_{a^{\prime}}$ and $g_{a b^{\prime}}$ have right-hand sides which are analytic functions of the coordinates $Y^{a}$, and are hence well defined everywhere on Lorentzian de Sitter spacetime [24].

\section{EVALUATION OF THE KERNEL}

In a de Sitter background the Ricci tensor is proportional to the metric through the cosmological constant: $R_{a b}=\Lambda g_{a b}$, and hence the formulae (10), (15), (18) and (19) lead to the following explicit expression for the solution of the inhomogeneous wave equation (8):

$$
\varphi_{c}(x)=\varphi_{c}^{(0)}(x)+2 \Lambda \int\left[\alpha\left(z\left(\mu\left(x, x^{\prime}\right)\right)\right) g_{c}^{a^{\prime}}+\beta\left(z\left(\mu\left(x, x^{\prime}\right)\right)\right) n_{c} n^{a^{\prime}}\right] \varphi_{a^{\prime}}\left(x^{\prime}\right) \sqrt{-\gamma\left(x^{\prime}\right)} d^{4} x^{\prime}
$$

where, from Eq. (24),

$$
\mu\left(x, x^{\prime}\right)=2 \rho \cos ^{-1} \sqrt{\frac{1}{2}\left(1+\frac{\eta_{a b} Y^{a}(x) Y^{b}\left(x^{\prime}\right)}{\rho^{2}}\right)},
$$

while Eqs. (18) and (19) should be exploited to express $\alpha$ and $\beta$, bearing in mind Eq. (26) jointly with

$$
z\left(x, x^{\prime}\right)=\frac{1}{2}\left[1+\cos \left(\frac{\mu\left(x, x^{\prime}\right)}{\rho}\right)\right] .
$$

Moreover, the bivector $g_{c}^{a^{\prime}}$ in the integrand (25) is given by [24]

$$
g_{a}^{b^{\prime}}=C^{-1}(\mu) \nabla_{a} n^{b^{\prime}}-n_{a} n^{b^{\prime}}, C(\mu)=-\frac{1}{\rho \sin (\mu / \rho)} .
$$

The right-hand side of the formula expressing $g_{a}^{b^{\prime}}$ is an analytic function of the coordinates $Y^{a}$ and is therefore well defined everywhere on de Sitter [24]. The integral on the right-hand side of Eq. (25) can be conveniently expressed the form

$$
f_{c}(x)=\int\left[\alpha(z) C^{-1}(\mu) \nabla_{c} \nabla^{a^{\prime}} \mu+(\beta(z)-\alpha(z))\left(\nabla_{c} \mu\right)\left(\nabla^{a^{\prime}} \mu\right)\right] \varphi_{a^{\prime}}\left(x^{\prime}\right) \sqrt{-\gamma\left(x^{\prime}\right)} d^{4} x^{\prime}
$$


with $\alpha$ and $\beta-\alpha$ given by (cf. (18) and (19))

$$
\begin{gathered}
\alpha(z)=\frac{(1+2 z)}{48 \pi^{2} \rho^{2}}\left[\frac{1}{z(1-z)}+\frac{1}{z^{2}} \log (1-z)\right], \\
\beta(z)-\alpha(z)=\frac{1}{48 \pi^{2} \rho^{2}}\left[\frac{\left(-3+2 z-2 z^{2}\right)}{z(1-z)}-\frac{3}{z^{2}} \log (1-z)\right] .
\end{gathered}
$$

Equation (25) is therefore an integral equation reading as

$$
\varphi_{c}(x)=\varphi_{c}^{(0)}(x)+\Lambda \int K_{c}^{a^{\prime}} \varphi_{a^{\prime}} \sqrt{-\gamma\left(x^{\prime}\right)} d^{4} x^{\prime}
$$

with unbounded kernel given by

$$
K_{c}^{a^{\prime}} \equiv 2\left[\alpha(z) C^{-1}(\mu) \nabla_{c} \nabla^{a^{\prime}} \mu+(\beta(z)-\alpha(z))\left(\nabla_{c} \mu\right)\left(\nabla^{a^{\prime}} \mu\right)\right]
$$

This kernel is indeed unbounded by virtue of the limits

$$
\begin{gathered}
48 \pi^{2} \rho^{2} \lim _{z \rightarrow 0} z \alpha(z)=\frac{1}{2}, \\
48 \pi^{2} \rho^{2} \lim _{z \rightarrow 1}(1-z) \alpha(z)=1, \\
48 \pi^{2} \rho^{2} \lim _{z \rightarrow 0} z(\beta(z)-\alpha(z))=-\frac{3}{2}, \\
48 \pi^{2} \rho^{2} \lim _{z \rightarrow 1}(1-z)(\beta(z)-\alpha(z))=-3 .
\end{gathered}
$$

At this stage, we can exploit (23) and (33) to re-express the kernel in the form

$$
\begin{aligned}
K_{c}^{a^{\prime}} & =\frac{\left(\nabla_{c} z\right)\left(\nabla^{a^{\prime}} z\right)}{24 \pi^{2} \rho^{4}(1-z)}\left[2+\left(-3+\frac{\sqrt{z}}{2}(1+2 z)\right)\left(\frac{1}{z(1-z)}+\frac{1}{z^{2}} \log (1-z)\right)\right] \\
& +\frac{\left(\nabla_{c} \nabla^{a^{\prime}} z\right)}{6 \pi^{2}} \sqrt{z}(1+2 z)\left[\frac{1}{z(1-z)}+\frac{1}{z^{2}} \log (1-z)\right] .
\end{aligned}
$$

Note now that $\varphi_{c}^{(0)}(x)$ in Eq. (32), being a solution of the homogeneous vector wave equation (11), admits the Huygens' principle representation [18]

$$
\varphi_{c}^{(0)}(x)=\int_{\Sigma^{\prime}} \sqrt{-\gamma\left(x^{\prime}\right)}\left[G_{c b^{\prime}} \varphi_{; m^{\prime}}^{(0) b^{\prime}}-G_{c b^{\prime} ; m^{\prime}} \varphi^{(0) b^{\prime}}\right] g^{m^{\prime} l^{\prime}} d \Sigma_{l^{\prime}}^{\prime},
$$

where, from Sec. 3 and the present section,

$$
\begin{gathered}
G_{c b^{\prime}}=\alpha g_{c b^{\prime}}+\beta \mu_{;} \mu_{; b^{\prime}}=\frac{1}{2} K_{c b^{\prime}} \\
G_{c b^{\prime} ; m^{\prime}}=\frac{1}{2} K_{c b^{\prime} ; m^{\prime}} .
\end{gathered}
$$


Unlike the work in [18], we here advocate the use of the Green function (15) rather than the sum, over all distinct geodesics between $x$ and $x^{\prime}$, of the Hadamard functions. To lowest order in the cosmological constant $\Lambda$, Eq. (39) may be used to approximate the desired solution of Eq. (32) in the form

$$
\varphi_{c}(x)=\varphi_{c}^{(0)}(x)+\Lambda \int K_{c}^{a^{\prime}} \varphi_{a^{\prime}}^{(0)} \sqrt{-\gamma\left(x^{\prime}\right)} d^{4} x^{\prime}+\mathrm{O}\left(\Lambda^{2}\right) .
$$

Omitting indices for simplicity, the general algorithm for solving Eq. (32), here re-written in the form

$$
\varphi=\varphi^{(0)}+\Lambda \int K \varphi
$$

would be instead

$$
\begin{gathered}
\varphi_{1}=\varphi^{(0)}+\Lambda \int K \varphi^{(0)} \\
\varphi_{2}=\varphi^{(0)}+\Lambda \int K \varphi_{1}=\varphi^{(0)}+\Lambda \int K \varphi^{(0)}+\Lambda^{2} \iint K K \varphi^{(0)} \\
\varphi_{n}=\varphi^{(0)}+\sum_{j=1}^{n} \Lambda^{j} \int \ldots \int K^{j} \varphi^{(0)} \\
\varphi=\lim _{n \rightarrow \infty} \varphi_{n} .
\end{gathered}
$$

\section{SEPARATION OF VARIABLES FOR THE VECTOR WAVE EQUATIONS}

Consider now the de Sitter metric in standard spherical coordinates

$$
d s^{2}=-f d t^{2}+\frac{1}{f} d r^{2}+r^{2}\left(d \theta^{2}+\sin ^{2} \theta d \phi^{2}\right)
$$

where $f \equiv 1-H^{2} r^{2}$ and $H$ is the Hubble constant. This metric satisfies the vacuum Einstein

equations with nonvanishing cosmological constant $\Lambda$ such that $H^{2}=\frac{\Lambda}{3}$. Moreover, the timelike unit normal vector fields $n$ to the $t=$ constant hypersurfaces

$$
n=\frac{\partial}{\partial t}
$$

form a geodesic and irrotational congruence. The 3 -metric induced on the $t=$ constant hypersurfaces turns out to be conformally flat, and the $(1,1)$ form of the spacetime Ricci tensor is simply given by

$$
R_{a}^{b}=3 H^{2} \delta_{a}^{b}
$$


From the previous sections it is clear we are interested in the generalized wave equation

$$
-\square X_{a}+\epsilon R_{a}^{b} X_{b}=\left(-\square+\epsilon \frac{R}{4}\right) X_{a}=0
$$

where $\epsilon \equiv \pm 1$. By virtue of the spherical symmetry of de Sitter spacetime these equations should be conveniently wtitten by using the expansion of $X$ in vector harmonics [25, 26, 27]. Following Zerilli [28] we have

$$
\begin{aligned}
X & =Y(\theta) e^{-i(\omega t-m \phi)}\left[f_{0}(r) d t+f_{1}(r) d r\right] \\
& +e^{-i(\omega t-m \phi)}\left[-\frac{m r}{\sin \theta} f_{2}(r) Y(\theta)+f_{3}(r) \frac{d Y}{d \theta}\right] d \theta \\
& +i e^{-i(\omega t-m \phi)}\left[-r \sin \theta f_{2}(r) \frac{d Y}{d \theta}+m f_{3}(r) Y(\theta)\right] d \phi,
\end{aligned}
$$

with $Y(\theta)$ solution of the spherical harmonics equation

$$
\left[\frac{d^{2}}{d \theta^{2}}+\cot \theta \frac{d}{d \theta}+\left(-\frac{m^{2}}{\sin ^{2} \theta}+L\right)\right] Y=0
$$

with $L \equiv l(l+1)$. These equations lead to a system of coupled ordinary differential equations for the functions $f_{0}, f_{1}, f_{3}$, besides a decoupled equation for $f_{2}$ ( $f_{2}$ being related to the transverse part of $X$ ). Indeed, for $l \neq 0,1$ (the latter case being trivial), we have

$$
\begin{aligned}
\frac{d^{2} f_{0}}{d r^{2}} & =-\frac{2}{r} \frac{d f_{0}}{d r}-\frac{1}{r^{2} f^{2}}\left[\omega^{2} r^{2}-L f+3 f(1-f)(1-\epsilon)\right] f_{0}+\frac{2 i \omega(f-1)}{r f} f_{1}, \\
\frac{d^{2} f_{1}}{d r^{2}} & =\frac{2(2-3 f)}{r f} \frac{d f_{1}}{d r}-\frac{1}{r^{2} f^{2}}\left[\omega^{2} r^{2}-L f-3 \epsilon f(1-f)+f(f-3)\right] f_{1} \\
& -\frac{2 i \omega(1-f)}{r f^{3}} f_{0}-\frac{2 L}{r^{3} f} f_{3}, \\
\frac{d^{2} f_{2}}{d r^{2}} & =\frac{2(1-2 f)}{r f} \frac{d f_{2}}{d r}-\frac{1}{r^{2} f^{2}}\left[\omega^{2} r^{2}-L f+f(1-f)(1-3 \epsilon)\right] f_{2}, \\
\frac{d^{2} f_{3}}{d r^{2}} & =\frac{2(1-f)}{r f} \frac{d f_{3}}{d r}-\frac{1}{r^{2} f^{2}}\left[\omega^{2} r^{2}-L f+3 f(1-f)(1-\epsilon)\right] f_{3}-\frac{2}{r} f_{1} .
\end{aligned}
$$

Equation (56) for $f_{2}(r)$ can be easily integrated in terms of hypergeometric functions. In fact, assuming

$$
f_{2}(r)=f^{-i \omega /(2 H)} \psi(r)
$$

the resulting equation for $\psi$ reads as

$$
\frac{d^{2} \psi}{d r^{2}}=-\frac{2 i}{r f}\left(2 i H^{2} r^{2}-i+\omega H r^{2}\right) \frac{d \psi}{d r}-\frac{1}{r^{2} f^{2}}\left[\omega^{2} r^{2}-L+(1-3 \epsilon) H^{2} r^{2}+3 i \omega H r^{2}\right]
$$


the solution of which is, in general, of the form

$$
\psi(r)=C_{1} r_{2}^{l} F_{1}\left(a_{-}, a_{+}, \frac{3}{2}+l, H^{2} r^{2}\right)+C_{2} r_{2}^{-1-l} F_{1}\left(a_{+}, a_{-}, \frac{1}{2}-l, H^{2} r^{2}\right),
$$

where we have defined

$$
a_{ \pm} \equiv-\frac{1}{4}\left[\frac{2 i \omega}{H}-3-2 l \pm(13-12 \epsilon)^{1 / 2}\right]
$$

Thus, when $\epsilon=1$, which corresponds to studying the vector wave equation (7), one finds

$$
a_{ \pm}=-\frac{1}{4}\left(\frac{2 i \omega}{H}-3-2 l \pm 1\right)
$$

whereas on taking $\epsilon=-1$, i.e. our consistency Eq. (8), one gets

$$
a_{ \pm}=-\frac{1}{4}\left(\frac{2 i \omega}{H}-3-2 l \pm 5\right) .
$$

The Lorenz gauge condition $\nabla_{\alpha} X^{\alpha}=0$, which only supplements Eq. (7), reduces instead to

$$
f_{3}=\frac{r^{2} f}{L} \frac{d f_{1}}{d r}-\frac{2 r(1-2 f)}{L} f_{1}+\frac{\omega r^{2} i}{L f} f_{0}
$$

The latter condition can be used, in principle, to obtain closed-form solutions of the various $f_{0}(r), f_{1}(r), f_{3}(r)$.

\section{WAVE EQUATION FOR METRIC PERTURBATIONS}

Although the vector wave equations in de Sitter spacetime are already considerably involved, the final step consists in studying the invertible wave operator $P_{a b}{ }^{c d}$ on metric perturbations. On considering the DeWitt supermetric

$$
E^{a b c d} \equiv \gamma^{a(c} \gamma^{d) b}-\frac{1}{2} \gamma^{a b} \gamma^{c d}
$$

the de Donder gauge in Eq. (4) can be re-expressed in the form

$$
\Phi_{a}(h)=E_{a}^{b c d} \nabla_{b} h_{c d}
$$

and the resulting Lichnerowicz operator [29], [30] on metric perturbations, obtained by expansion of the Einstein-Hilbert action to quadratic order in $h_{a b}$, subject to $\Phi_{a}(h)=0$, reads as [31]

$$
P_{a b}^{c d} \equiv E_{a b}^{c d}(-\square+R)-2 E_{a b}^{l f} R_{l h f}^{c} \gamma^{d h}-E_{a b}^{l d} R_{l}^{c}-E_{a b}^{c l} R_{l}^{d}
$$


A wave equation for metric perturbations is therefore given by (see the Introduction)

$$
P_{a b}{ }^{c d} h_{c d}=0
$$

where [31]

$$
\begin{aligned}
P_{a b}^{c d} & =E_{a b}^{c d}(-\square+R)-2 E_{a b}^{l f} R_{l h f}^{c} \gamma^{d h}-E_{a b}^{l d} R_{l}^{c}-E_{a b}^{c l} R_{l}^{d} \\
& =E_{a b}{ }^{c d}(-\square+R)-2 E_{a b}^{l f} R_{m l}{ }^{d n} \gamma^{m c} \gamma_{n f}-\frac{R}{4} E_{a b}{ }^{c d}-\frac{R}{4} E_{a b}{ }^{c d} \\
& =E_{a b}{ }^{c d}(-\square+R)-\frac{R}{6} E_{a b}^{l f} \delta_{m l}^{d n} \gamma^{m c} \gamma_{n f}-\frac{R}{2} E_{a b}{ }^{c d} \\
& =E_{a b}{ }^{c d}\left(-\square+\frac{1}{2} R\right)-\frac{R}{6} E_{a b}^{l f} \delta_{m l}^{d n} \gamma^{m c} \gamma_{n f} \\
& =E_{a b}{ }^{c d}\left(-\square+\frac{1}{2} R\right)+\frac{R}{6} E_{a b}^{c d}+\frac{R}{6} \gamma_{a b} \gamma^{c d} \\
& =E_{a b}{ }^{c d}\left(-\square+\frac{2}{3} R\right)+\frac{R}{6} \gamma_{a b} \gamma^{c d} .
\end{aligned}
$$

The wave equation then becomes

$$
0=P_{a b}^{c d} h_{c d}=\left(-\square+\frac{2}{3} R\right) \bar{h}_{a b}+\frac{R}{6} \gamma_{a b} h
$$

or

$$
\left(-\square+\frac{2}{3} R\right) \bar{h}_{a b}-\frac{R}{6} \gamma_{a b} \bar{h}=0,
$$

implying also

$$
\left(-\square+\frac{2}{3} R\right) \bar{h}-\frac{2}{3} R \bar{h}=0,
$$

that is

$$
\square \bar{h}=0,
$$

after contraction with $\gamma^{a b}$. 


\section{A. Even metric perturbations}

Metric perturbations of even parity can be written in the form

$$
\begin{aligned}
& h_{00}=f e^{-i(\omega t-m \phi)} H_{0}(r) Y(\theta), \\
& h_{01}=e^{-i(\omega t-m \phi)} H_{1}(r) Y(\theta) \text {, } \\
& h_{02}=e^{-i(\omega t-m \phi)} h_{0}(r) \frac{d Y}{d \theta} \text {, } \\
& h_{03}=i m e^{-i(\omega t-m \phi)} h_{0}(r) Y(\theta) \text {, } \\
& h_{11}=\frac{1}{f} e^{-i(\omega t-m \phi)} H_{2}(r) Y(\theta) \text {, } \\
& h_{12}=e^{-i(\omega t-m \phi)} h_{1}(r) \frac{d Y}{d \theta}, \\
& h_{13}=i m e^{-i(\omega t-m \phi)} h_{1}(r) Y(\theta) \text {, } \\
& h_{22}=r^{2} e^{-i(\omega t-m \phi)}\left[K(r) Y(\theta)+G(r) \frac{d^{2} Y}{d \theta^{2}}\right] \text {, } \\
& h_{23}=i m r^{2} G(r) e^{-i(\omega t-m \phi)}\left[\frac{d Y}{d \theta}-\cot \theta Y(\theta)\right] \text {, } \\
& h_{33}=r^{2} e^{-i(\omega t-m \phi)}\left\{K(r) \sin ^{2} \theta Y(\theta)\right. \\
& \left.+G(r)\left[-m^{2} Y(\theta)+\sin \theta \cos \theta \frac{d Y}{d \theta}\right]\right\} \text {. }
\end{aligned}
$$


The wave equation (68) leads to the following system of coupled differential equations:

$$
\begin{aligned}
& \frac{d^{2} H_{0}}{d r^{2}}=\frac{2}{r f}(2-3 f) \frac{d H_{0}}{d r}-\frac{1}{r^{2} f^{2}}\left[\omega^{2} r^{2}+2(1-f)(1-4 f)-f L\right] H_{0} \\
& +\frac{2 H^{2} r}{f} \frac{d H_{2}}{d r}-\frac{4 H^{2} r}{f} \frac{d K}{d r}+\frac{2 H^{2} r L}{f} \frac{d G}{d r} \\
& +\frac{2 H^{2} L}{f} G-\frac{4 H^{2} L}{r f} h_{1}-\frac{2 H^{2}(1-6 f)}{f^{2}} H_{2}-\frac{4 H^{2}}{f} K, \\
& \frac{d^{2} H_{1}}{d r^{2}}=\frac{2}{r f}(1-2 f) \frac{d H_{1}}{d r}-\frac{1}{r^{2} f^{2}}\left[\omega^{2} r^{2}-L f-2\left(2-f^{2}\right)\right] H_{1} \\
& -\frac{2 L}{f r^{3}} h_{0}-\frac{2 i \omega r H^{2}}{f^{2}}\left(H_{2}+H_{0}\right) \\
& \frac{d^{2} H_{2}}{d r^{2}}=\frac{2(2-3 f)}{r f} \frac{d H_{2}}{d r}-\frac{1}{r^{2} f^{2}}\left[\omega^{2} r^{2}-L f-10+6 f+12(1-f)^{2}\right] H_{2} \\
& +\frac{2 H^{2} r}{f} \frac{d H_{0}}{d r}-\frac{4 H^{2} r}{f} \frac{d K}{d r}+\frac{2 r L H^{2}}{f} \frac{d G}{d r} \\
& +\frac{2 L(3-2 f)}{r^{2} f} G-\frac{4 L}{r^{3} f} h_{1}-\frac{4(3-2 f)}{r^{2} f} K-\frac{2 H^{2}(1-2 f)}{f^{2}} H_{0}, \\
& \frac{d^{2} h_{0}}{d r^{2}}=-\frac{1}{r^{2} f^{2}}\left[\omega^{2} r^{2}-L f-4 f(1-f)\right] h_{0}-\frac{2 i \omega r H^{2}}{f} h_{1}-\frac{2}{r} H_{1}, \\
& \frac{d^{2} h_{1}}{d r^{2}}=\frac{6 r H^{2}}{f} \frac{d h_{1}}{d r}-\frac{1}{r^{2} f}\left[\omega^{2} r^{2}-L f+10(1-f)^{2}-6+2 f\right] h_{1}+\frac{1}{r f^{2}}[2-(1+f) L] G \\
& -\frac{1+f}{r f^{2}} H_{2}+\frac{2}{r f} K+\frac{H^{2} r}{f^{2}} H_{0} \\
& \frac{d^{2} G}{d r^{2}}=\frac{2}{r f}(1-2 f) \frac{d G}{d r}-\frac{1}{r^{2} f^{2}}\left[\omega^{2} r^{2}-L f+2 f(3 f-2)\right] G-\frac{4 h_{1}}{r^{3}}, \\
& \frac{d^{2} K}{d r^{2}}=\frac{2(1-2 f)}{r f} \frac{d K}{d r}-\frac{1}{r^{2} f^{2}}\left[\omega^{2} r^{2}-L f-2 f(2-f)\right] K-\frac{2 L}{r^{2}} G \\
& -\frac{2}{r^{2} f} H_{2}+\frac{2 H^{2}}{f} H_{0}
\end{aligned}
$$

To these equations one should add the de Donder gauge components

$$
\begin{aligned}
\frac{d H_{1}}{d r}= & \frac{i \omega L}{2 f} G+\frac{L}{r^{2} f} h_{0}-\frac{i \omega}{2 f} H_{2}-\frac{i \omega}{f}\left(K+\frac{H_{0}}{2}\right)+\frac{2(1-2 f)}{r f} H_{1}, \\
\frac{d H_{2}}{d r}= & \frac{2(1-3 f)}{r f} H_{2}-\frac{d H_{0}}{d r}+2 \frac{d K}{d r}-L \frac{d G}{d r}-\frac{2 L}{r} G \\
& +\frac{2 L}{r^{2}} h_{1}+\frac{4}{r} K+\frac{2 H^{2} r}{f} H_{0}-\frac{2 i \omega}{f} H_{1}, \\
\frac{d h_{1}}{d r}= & \frac{2(1-2 f)}{r f} h_{1}+\frac{L-2}{2 f} G-\frac{i \omega}{f^{2}} h_{0}+\frac{1}{2 f}\left(H_{2}-H_{0}\right) .
\end{aligned}
$$

Metric perturbations of odd parity are instead found to vanish identically. 


\section{CONCLUDING REMARKS}

A consistency analysis for gravitational waves in curved background is not, by itself, new in the literature and it has been outlined, for example, in Sec. II and Appendix B of Ref. [32]. However, that paper was mainly concerned with gravitational instability in higher dimensions. In this paper, we have endeavoured to provide explicit solution formulae for the covector which solves the residual gauge problem expressed by Eq. (6), and this has been accomplished in Secs. 4 and 5. Section 6 has then moved on to work out all equations obeyed by metric perturbations subject to the de Donder gauge in the de Sitter spacetime. Although the system (74)-(76) obeyed by metric perturbations looks very complicated, our approach is suitable for computer-assisted investigation of such equations, with the possibility of investigating gravitational waves in inflationary cosmology [23] and/or other

curved backgrounds relevant for strong-gravity regimes. In particular, as discussed in [33], a significant fraction of energy, in the form of a stochastic background of gravitational waves could emerge during the reheating after inflation. In this situation, the exact classification of gravitational wave solutions (as in Sec. 6) could be crucial in order to discriminate among the various signals. In a forthcoming paper, starting from the presented solutions, we will discuss the problem of generation and production of gravitational waves in the de Sitter background [34].

\section{APPENDIX A: BIVECTORS AND BISCALARS}

In Eq. (13), $g_{c^{\prime}}^{a}$ is the geodesic parallel displacement bivector (in general, bitensors behave as a tensor both at $x$ and at $x^{\prime}$ ) which effects parallel displacement of vectors along the geodesic from $x^{\prime}$ to $x$. In general, it is defined by the differential equations

$$
\sigma^{; b} g_{c^{\prime} ; b}^{a}=\sigma^{; b^{\prime}} g_{c^{\prime} ; b^{\prime}}^{a}=0
$$

jointly with the coincidence limit

$$
\lim _{x^{\prime} \rightarrow x} g_{c^{\prime}}^{a}=\left[g_{c^{\prime}}^{a}\right]=\delta_{c}^{a}
$$

The bivector $g_{c^{\prime}}^{a}$, when acting on a vector $B^{c^{\prime}}$ at $x^{\prime}$, gives therefore the vector $\bar{B}^{a}$ which is obtained by parallel transport of $B^{c^{\prime}}$ to $x$ along the geodesic connecting $x$ and $x^{\prime}$, i.e.

$$
\bar{B}^{a}=g_{c^{\prime}}^{a} B^{c^{\prime}}
$$


In Eq. (14), $\triangle\left(x, x^{\prime}\right)$ is a biscalar built from the Van Vleck-Morette determinant

$$
D\left(x, x^{\prime}\right) \equiv \operatorname{det}\left(\sigma_{; a b^{\prime}}\right)
$$

according to

$$
\triangle\left(x, x^{\prime}\right) \equiv \frac{1}{\sqrt{-\gamma(x)}} D\left(x, x^{\prime}\right) \frac{1}{\sqrt{-\gamma\left(x^{\prime}\right)}} .
$$

The biscalar $\triangle\left(x, x^{\prime}\right)$ has unit coincidence limit: $[\triangle]=1$; as a function of $x$ (resp. $\left.x^{\prime}\right)$, it becomes infinite on any caustic formed by the geodesics emanating from $x^{\prime}$ (resp. $x$ ). When $\triangle$ diverges in this way, $x$ and $x^{\prime}$ are said to be conjugate points [35].

\section{ACKNOWLEDGMENTS}

G. Esposito is grateful to the Dipartimento di Scienze Fisiche of Federico II University, Naples, for hospitality and support, and to CNR for financial support.

[1] A. Abramovici et al., Science 256 (1992) 325; http:// www.ligo.org

[2] B. Caron et al., Class. Quantum Grav. 14 (1997) 1461; http:// www.virgo.infn.it

[3] H. Luck and the GEO600 Team, Class. Quantum Grav. 14 (1997) 1471; http:// www.geo600.uni-hannover.de

[4] M. Ando et al. (TAMA Collaboration), Phys. Rev. Lett. 86 (2001) 3950; http:// tamago.mtk.nao.ac.jp

[5] A. Buonanno and Y. Chen, Phys. Rev. D 64 (2001) 042006.

[6] A. Buonanno and Y. Chen, Phys. Rev. D 69 (2004) 102004.

[7] D. Babusci, L. Baiotti, F. Fucito and A. Nagar, Phys. Rev. D 64 (2001) 062001.

[8] S. Capozziello and M. Francaviglia, Gen. Rel. Grav. 40 (2008) 357.

[9] S. Capozziello, M. De Laurentis and M. Francaviglia, Astrop. Phys. 29 (2008) 125.

[10] http://www.lisa-science.org

[11] http://www.rssd.esa.int/Planck

[12] F. A. Jenet, Astroph. Jou. 653 (2006) 1571.

[13] http:// www.skatelescope.org

[14] W. L. S. de Paula, O. D. Miranda and R.M. Marinho, Class. Quantum Grav. 21 (2004) 4595. 
[15] S. L. Dubovsky, P. G. Tinyakov and I. I. Tkachev, Phys. Rev. Lett. 94 (2005) 181102.

[16] L. Lorenz, Phil. Mag. 34 (1867) 287.

[17] F. G. Friedlander, The Wave Equation on a Curved Space-Time (Cambridge University Press, Cambridge, 1975).

[18] B. S. DeWitt and R. W. Brehme, Ann. Phys. (N.Y.) 9 (1960) 220.

[19] G. Bimonte, E. Calloni, L. Di Fiore, G. Esposito, L. Milano and L. Rosa, Class. Quantum Grav. 21 (2004) 647.

[20] H. S. Ruse, Proc. Lond. Math. Soc. 32 (1931) 87.

[21] J. L. Synge, Proc. Lond. Math. Soc. 32 (1931) 241.

[22] J. L. Synge, Relativity: The General Theory (North-Holland, Amsterdam, 1960).

[23] S. W. Hawking, T. Hertog and N. Turok, Phys. Rev. D 62 (2000) 063502.

[24] B. Allen and T. Jacobson, Commun. Math. Phys. 103 (1986) 669.

[25] E. M. Lifshitz and I. M. Khalatnikov, Adv. Phys. 12 (1963) 185.

[26] U. H. Gerlach and U. K. Sengupta, Phys. Rev. D 18 (1978) 1773.

[27] R. T. Jantzen, J. Math. Phys. 19 (1978) 1163.

[28] F. J. Zerilli, Phys. Rev. D 9 (1974) 860.

[29] A. Lichnerowicz, Bull. Soc. Math. France 92 (1964) 11.

[30] G. W. Gibbons and M. J. Perry, Nucl. Phys. B 146 (1978) 90.

[31] I. G. Moss, Quantum Theory, Black Holes and Inflation (John Wiley \& Sons, New York, 1996).

[32] G. Gibbons and S. A. Hartnoll, Phys. Rev. D 66 (2002) 064024.

[33] J. Garcia-Bellido, D. G. Figueroa and A. Sastre, Phys. Rev. D 77 (2008) 043517.

[34] D. Bini, S. Capozziello, G. Esposito and M. De Laurentis, in preparation.

[35] B.S. DeWitt in Relativity, Groups and Topology II, eds. B.S. DeWitt and R. Stora (NorthHolland, 1984). 